\title{
The Origins of Counting Algorithms
}

\author{
Jessica F. Cantlon ${ }^{a}$, Steven T. Piantadosia ${ }^{a}$, Stephen Ferrigno ${ }^{a}$, Kelly D. Hughes ${ }^{a}$, and \\ Allison M. Barnarda \\ aBrain \& Cognitive Sciences, University of Rochester, New York, Meliora Hall Box 270268, \\ Rochester, NY 14627
}

\begin{abstract}
Humans' ability to 'count' by verbally labeling discrete quantities is unique in animal cognition. The evolutionary origins of counting algorithms are not understood. We report that non-human primates exhibit a cognitive ability that is algorithmically and logically similar to human counting. Monkeys were given the task of choosing between two food caches. Monkeys saw one cache baited with some number of food items, one item at a time. Then, a second cache was baited with food items, one at a time. At the point when the second set approximately outnumbered the first set, monkeys spontaneously moved to choose the second set even before it was completely baited. Using a novel Bayesian analysis, we show that monkeys used an approximate counting algorithm to increment and compare quantities in sequence. This algorithm is structurally similar to formal counting in humans and thus may have been an important evolutionary precursor to human counting.
\end{abstract}

\section{Keywords}

counting; mathematical cognition; animal cognition; cognitive development; non-human primate

Human counting affords a kind of condition-controlled logic wherein counters can increment a set by labeling items "one, two, three, four..." until some condition is met. For example, items can be incremented until all items are counted, until the number of items counted reaches a target number, or until the items counted outnumber the value of another set. This study asks whether this condition-controlled logic, inherent in human counting, is a feature of nonhuman quantitative reasoning. Evidence that non-human animal quantification includes logic that is inherent in human counting would provide a new theoretical basis for connecting the principles of human counting with the principles of evolutionarily primitive quantity systems.

Non-human animals do not use words like "one, two, three" or numerals like " $1,2,3$ " to 'count' in the way that humans do when they count. Nonetheless, it is well-established that monkeys and other animals can approximate quantities without these symbolic labels (Gallistel, 1989; Gallistel \& Gelman, 1992). For example, research studies using computerized tasks have shown that monkeys can roughly determine which of two sets of

Corresponding author: Jessica Cantlon, University of Rochester, Brain \& Cognitive Sciences, Meliora Hall Box 270268, Rochester, NY 14627, 585-276-5099, jcantlon@bcs.rochester.edu. 
dots has the larger number (Beran, 2007; Brannon \& Terrace, 1998; Cantlon \& Brannon, 2006). Other studies have shown that apes and monkeys compute simple addition outcomes (Beran, 2001; Cantlon \& Brannon, 2007). For example, monkeys can discriminate that when three objects are combined with five more, the total number is 8 , not 2 or 4 . Monkeys also have been shown to discriminate quantities spontaneously, in naturalistic foraging tasks. Semi-wild and experiment-naïve primates can choose the larger of two sets of food items without any prior exposure to quantity decision tasks (Barnard et al., 2013; Flombaum, Junge, \& Hauser, 2005; Hauser, Carey, \& Hauser, 2000). Many animal species, including even birds and fish, estimate quantity (Agrillo, Dadda, \& Bisazza, 2007; Emmerton, 2001; Pepperberg, 2006). A non-symbolic sense of approximate quantity is likely a fundamental component of animal cognition (Gallistel, 1989).

The basic quantity skills of non-human animals are comparable to some of the numerical skills that human infants and young children exhibit in experiments on pre-linguistic human mathematical concepts (Brannon, 2002; Lipton \& Spelke, 2003; Wynn, 1992; Xu \& Spelke, 2000). For example, 6-month-old human infants are surprised (look longer) when a set of 5 objects is combined with a set of 5 objects behind a screen and then the screen is lifted to reveal only 5 objects compared to when 10 objects are revealed (Wynn, 1992; McCrink \& Wynn, 2004). Thus infants, children, and non-human animals possess cognitive mechanisms for representing and operating on numerical values. However, unlike non-humans, human children go on to learn a verbal counting routine. An area that has not been well explored is to what extent non-humans possess the logical operations that form the basis of verbal counting. Although some studies have shown that, with training, monkeys can compare sequentially presented sets (Beran et al., 2014; Jordan et al., 2008), and other studies have shown that, with training, animals can associate quantitative meanings with numerals or words (Boysen \& Bernston, 1989; Pepperberg, 2006; Tomonaga \& Matsuzawa, 2002), none have defined a formal, logical algorithm of sequential quantification in non-human animals.

All current formalizations of non-symbolic quantification assume that mental comparison happens at the end of incrementing, not item-by-item (Dehaene, 2009; Meck \& Church, 1983). However, this assumption is based on an absence of data rather than a positive argument that comparison occurs only after all items are incremented. Other theories from the animal learning literature argue that non-human animals represent a conditioned gradient of reinforcement across sets of items to discriminate quantity (eg., Skinner \& Ferster, 1957; Mechner, 1958). No studies have tested whether non-human animals spontaneously compare the relative values of two sets as they are in the process of quantifying. This question is important because it will determine to what extent the primitive quantity routines of nonhuman animals contain logical elements of human counting - an issue central to discovering the evolutionary origins of human counting.

Here we show that monkeys spontaneously compare a remembered quantity to item-by-item changes in the value of a new quantity, and thus keep constant tabs on the relative values of sets by incrementally comparing them. Furthermore, using a novel Bayesian data analysis, we show that the monkeys' spontaneous behavior is explained by a cognitive algorithm that is algorithmically and logically similar to human counting. 


\section{Methods}

Monkeys (N=2, Papio anubis) were presented with a food choice task in which pieces of food (shelled peanuts) were sequentially placed into two food caches that were spatially separated by at least an arm's length (Figure 1). Each food cache was baited with a different quantity that ranged from 1 to 8 food items. The food caches were baited consecutively such that all food items were placed one-by-one into the first food cache before the second food cache was baited one-by-one. After the two caches were baited, the animal was permitted to choose between the two caches and indicated her choice by touching it. Upon making a choice, the animal was given the contents of her chosen food cache. Two control conditions were tested to demonstrate that the animals' quantity choices were not determined by 1) experimenter cueing or 2) the temporal duration of the stimulus presentation.

\section{Subjects}

Two olive baboons (Papio anubis), housed and tested at the Seneca Park Zoo in Rochester, NY, participated in these experiments. Primate chow and fresh fruits and vegetables are provided every morning, and water is available ad libitum. Research with these subjects was approved by the Seneca Park Zoo Conservation \& Research Committee.

Subjects did not have prior laboratory training in quantity discrimination. At the time when these data were collected, both subjects were experiment-naïve: neither animal had participated in an experimental task other than the current task. In the current task, subjects are reinforced with food on every trial and thus are not conditioned to discriminate quantity.

Like many primate studies, two subjects provide a sufficient sample size for our goals. In contrast to most human research, our study is not aimed at making inferences about a population. Instead, our primary interest is in exploring whether it is possible for countinglike behavior to exist in non-human animals. Logically, a demonstration of possibility requires only a single example ${ }^{1}$. Thus, a small sample size is sufficient for determining whether a cognitive capacity is possible in a population. A small sample also is ideal for collecting hundreds and thousands of measurements from the same individuals over long periods of time, which can provide rich insights into cognitive processes. The limitation of a small sample size is that it licenses population-level inferences only if the population is mostly homogenous. Indeed, there are reasons to expect that non-human primates are homogenous in their cognitive capacity for quantitative reasoning because the ability to make quantitative judgments is phylogenetically widespread among animals (Gallistel, 1989) and thus is likely to appear in most individuals. However, the issue of whether or not our observations can be extrapolated to the population level is independent of our conclusion which claims that counting-like behavior is possible in a non-human animal.

\section{Apparatus}

The apparatus consisted of a small and short rectangular table $(75 \mathrm{~cm}$ long $\times 35 \mathrm{~cm}$ deep $\times$ $17 \mathrm{~cm}$ high). One long side of the table top was open so that an experimenter could work the

\footnotetext{
${ }^{1}$ For example, if we want to know whether it is possible for a woman to achieve grandmaster performance in chess, we need only look at the performance of Judit Polgar to see that it is.
} 
apparatus, but the other three sides were shielded by plexiglass $(30 \mathrm{~cm}$ high) to prevent baboons from interacting with the apparatus until the appropriate time. When in use, the long side of plexiglass was pushed flush with the mesh of an enclosure, a subject sat behind the plexiglass (and the mesh of the enclosure), and an experimenter sat opposite the subject. There were three equally spaced ports $(2.5 \mathrm{~cm}$ diameter, $30 \mathrm{~cm}$ apart $)$ in the plexiglass that subjects could use to indicate their choices.

All experimental manipulations were conducted on a sliding panel $(75 \mathrm{~cm}$ long $\times 17 \mathrm{~cm}$ deep) that sat atop the table. The purpose of this panel, which was the same length as the table, but only half as deep, was to control a subject's access to the experiment until the appropriate time. When the panel was close to the experimenter, the subject did not have access to the experimental items, however, when the panel was pushed forward, toward the subject, the subject could reach through a port in the plexiglass and indicate its choice. The contents of the panel were three identical, opaque, cardstock cylinders, placed upright on a circular end, each in front of one of the ports in the plexiglass shield. The cylinders were open on both circular ends so that the experimenter could drop items into a cylinder and also lift a cylinder up, leaving the contents of a cylinder on the panel. Once items were dropped into the cylinders, the items were hidden from a subject. The items to be enumerated were shelled half peanuts. A similar description of the apparatus and procedures is found in Barnard et al. (2013).

\section{Procedure}

Each session was conducted by two experimenters. One experimenter worked the apparatus, while a second experimenter recorded the choices made by the subject, monitored the first experimenter for trial accuracy, and also operated a video camera, which was used to record each session. Sessions were conducted when a subject could be temporarily isolated from the troop in an enclosure, which was between 1 and 3 times a week per monkey. Each session was approximately 30 minutes. In order to keep the animals motivated to participate in experiments and familiarized with the experimental task, they are tested continuously throughout the week. The minimum number of trials collected was the number of trials needed to detect above-chance performance in a binomial test.

Before testing began, the experimenters set up the apparatus: the plexiglass side of the table was placed flush with the subject's enclosure, the sliding panel was placed on the experimenter's side of the table, the three cylinders were in place on the panel, and one experimenter sat opposite the subject. Cylinders were baited sequentially, one item at a time. For example, if the experimenter was testing the numerical pair 3 versus 6 , the first 3 peanuts presented were baited into one cylinder one-at-a-time, and the following 6 peanuts were then baited into a second cylinder one-at-a-time. Again, the experimenter was careful to touch the cylinders in the same way and for the same amount of time. To initiate a trial, an experimenter showed the subject one peanut about $30 \mathrm{~cm}$ from the subject and above the experimental panel. Each peanut was presented to the subject for $2 \mathrm{~s}$ and then placed into a cylinder with a $2 \mathrm{~s}$ delay between items. To ensure that subjects were not basing their choices on the spatial location of the sets, the larger and smaller numerical values were equally likely to appear in any one of the three cylinders across the session. Note, that although there 
were always three cylinders on the board, only two of these cylinders were baited with food on each trial. The presence of the third cylinder allowed us to monitor subjects' understanding of the general task requirement that only baited cups should be chosen. Subjects almost never selected the empty cylinder ( $1 \%$ of trials) indicating that they understood the task.

After the cylinders had been baited with peanuts, the panel was pushed forward and the subject was allowed to make a choice from among the three cylinders. Experimenters did not look at the cups after baiting until after the subject indicated their choice. The subject indicated its choice by poking its finger through the port in front of the desired cylinder. Then, the experimenter removed the cylinder from over the desired food, and the food reward was fed, one peanut at a time, to the subject through the same port. In the case that there was no food reward under the chosen cylinder, the subject received no reward. When the subject had received the entirety of its reward, the experimenter removed the other two cylinders from the panel, revealing their contents. The experimenter removed the two sets of reward. Once all peanuts were removed from the board, the experimenter pulled the panel back to her side of the apparatus, and reset the board. The next trial was initiated. This procedure was used throughout the experiment.

Interspersed with the sequential trials, described above, were trials in which food items were presented simultaneously. On simultaneous trials, two food caches to be compared were presented all at once, one set in each hand, and then placed into their cylinders. The numerical values of the sets were the same as for the sequential trials (values of 1 to 8 items). Simultaneous trials were randomly intermixed with sequential trials across the experiment and comprised 50\% of the total trials (170 simultaneous trials for Monkey 1 and 194 for Monkey 2). The simultaneous trials are not the focus of our current analyses. However, both animals were highly accurate on the simultaneous trials (Mean Accuracy = 80\%; Monkey 1: 130/170, $\mathrm{p}<.001$; Monkey 2: 160/194, $\mathrm{p}<.001)$ and they showed a numerical ratio effect in their performance (Monkey $1: \mathrm{R}^{2}=.49$, Monkey $2: \mathrm{R}^{2}=.90$ ). We included simultaneous trials in each session so that subjects would not be biased to use the duration of presentation to make their discrimination -- since on simultaneous trials each set is presented for the same total duration.

\section{Main Experiment}

Subjects were tested on a total of approximately 175 sequential trials over 10-12 sessions. The number pairs tested were all possible pairs between 1 and 8 ( 27 different numerical comparisons). Subjects completed approximately 6 trials in each number pair. The order of the test trials was randomized within and between subjects. Importantly, subjects were reinforced on every trial in that they received whichever quantity of food items they chose. The only way that reinforcement could be construed as differential reinforcement is if the animals actively discriminated the quantities of the reward they received from the one they did not receive. Thus, the animals were not trained to discriminate quantities at any point in this experiment and the animals' discrimination abilities derive from their spontaneous quantitative cognition. 


\section{Experimenter Cueing Control Condition}

Immediately following the main experiment, a control condition was conducted to rule out the possibility that experimenter cueing led the animal to their quantity choices. If subjects relied on experimenter cues to make their choices during the main experiment then, they would fail to select the greater set once those cues were removed. In this condition one experimenter would fully bait the first cylinder, then a second experimenter would bait the second cylinder. Each experimenter read the numerical value to-be-baited from a trial list that only showed the quantity of one set. The two experimenters sat back-to-back so that they could not see each other. Thus, each experimenter only knew the value of the cup they baited and did not see the baiting of the other cup, eliminating the possibility of subconsciously cuing the animals.

Subjects were first familiarized with this new procedure with values of 1 vs. 2 and 2 vs. 9 . Both subjects performed above chance on the first session with this new procedure (Binomial tests; Monkey 1: 19/24, p <.01; Monkey 2: 17/24, p <.05). Subjects were tested with 1 vs. 2 and 2 vs. 9 until they reached 70\% accuracy for two sessions ( 24 trials/session). Monkey 1 required the minimum 2 sessions to reach criterion and Monkey 2 required 4 sessions. Subjects then were tested on approximately 100 additional trials of this condition. The number pairs tested were: 1 vs. 5,1 vs. 6,1 vs. 7,1 vs. 8,2 vs. 5,2 vs. 6,2 vs. 7,2 vs. 8,3 vs. 5,3 vs. 6,3 vs. 7,3 vs. 8,4 vs. 5,4 vs. 6,4 vs. 7,4 vs. 8 . Subjects completed approximately 6 trials in each number pair. The order of the test trials was randomized within and between subjects.

\section{Timing Control Condition}

A second control condition, tested after the cueing control sessions, was conducted to rule out the possibility that the relative duration of the two sets determined the animals' choices. If subjects relied on timing cues to make their choices during the main experiment then, they would fail to select the greater set once those cues were removed. As mentioned above, subjects did not likely use the relative duration of the baiting of the two sets as a cue to the larger amount since a subset of the trials of the main experiment presented both sets simultaneously, where each set was presented for the same total duration, and the animals successfully discriminated the number of items in the sets. Nonetheless, we conducted an additional control condition to rule out the possibility that animals were using total duration as a cue during the sequential trials. In this condition, the duration of baiting time of one of the cylinders was 30s. On half of the trials the set with the larger numerical value had the 30s baiting time and on the remaining half of trials the smaller quantity had the 30s baiting time. The 30s presentation time in the control condition was longer than the typical baiting time for any of the quantities tested (Max typical baiting time was 24s for 6 items).

Subjects were tested on a total of 48 trials in this condition over two sessions: 24 control trials randomly intermixed with 24 trials of the standard baiting time protocol from the main experiment (ie., $4 \mathrm{~s}$ per item for both sets). The number pairs tested on control trials were: 2 vs. 4.2 vs. 6,4 vs. 6 . Subjects completed 8 trials per number pair in each of the standard and control timing conditions. The order of the test trials was randomized within and between subjects. 


\section{Data coding}

The animals' choices were coded offline by two independent coders (inter-rater reliability; Kappa Test: $\kappa=.875, \mathrm{p}<.001)$. A small percentage of trials were not included in the coding for switches due to the monkey not sitting at the first cylinder (15\%), dark or no video (7\%), ambiguous switch points (5\%), or the monkey switching back to the $1^{\text {st }}$ cup (2\%). For the switching behavior analyses, this left a total of 111 sequential trials for Monkey 1 and 137 for Monkey 2 in the main experiment, 108 for Monkey 1 and 99 for Monkey 2 in the cueing control condition, and 44 for Monkey 1 and 41 Monkey 2 in the timing control condition.

\section{Results}

Both monkeys chose the larger quantity on the majority of trials [Mean Accuracy $=68 \%$, Monkey 1: 81/111, p < 0.001, Monkey 2: 86/137, p < 0.001]. As expected, the monkeys' discrimination abilities were modulated by the numerical ratio between the choices (Figure 2). The ratio effect is a common characteristic of non-symbolic numerical discrimination known as Weber's Law (Gallistel \& Gelman, 2000). Figure 2 shows that the monkeys' actual accuracy fits the predicted accuracy under Weber's law (Monkey $1: \mathrm{R}^{2}=.98$, Monkey 2: $\mathrm{R}^{2}=.72$; following Cantlon \& Brannon, 2007; Pica, Lemer, Izard, \& Dehaene, 2004). The monkeys' average sensitivity to differences between numerical values was .86 (their weber fraction). This means that the monkeys required nearly a $2: 1$ ratio between quantities to reliably identify the larger of the two quantities. Prior research on quantity discrimination has reported finer weber fractions in non-human primates (eg., Cantlon \& Brannon, 2006; Nieder \& Miller, 2003). In contrast to prior research, subjects in the current study had no prior training with quantity discrimination and were rewarded with food on every trial due to the nature of this food choice paradigm. Differences in experience and motivation could explain differences in quantity discrimination across studies.

A more surprising characteristic of the monkeys' behavior occurred in the moments leading up to their final quantity choice. On $37.5 \%$ of trials, the animal physically moved from the first set to the second set before the experimenter had finished baiting the second set. There was no requirement in this task for the animals to make a speeded decision and thus their switching behavior was based on spontaneous online decision-making. A video of this midbaiting "switching" behavior in monkeys is available for viewing online (Supplementary Video 1). These spontaneous mid-baiting "switches" were generally logical in that they largely occurred when the second set was larger than the first set (Average $=70 \%$ logical, St. Dev. $=4 \%, 64 / 93, \mathrm{p}<.001 ;$ Monkey 1: 24/33, $\mathrm{p}<.01$; Monkey 2: 40/60, $\mathrm{p}<.01)$. This result suggests that the animals were spontaneously switching to Set 2 based on the relative value of Set 2 compared to Set 1.

We analyzed the point in the baiting sequence that the baboon physically moved from Set 1 to Set 2, or "switched", as a function of the relative value of Set 2 compared to Set 1. First, we found that monkeys showed an increasing probability of switching as the relative value of Set 2 to Set 1 increased (Figure 3). On each trial, for each item added to Set 2, we coded whether or not the animal had switched by that item. We plotted the proportion of switches as a function of the relative value of Set 2 to Set 1 at each item added to Set 2 (Figure 3, xaxis). The data show that as Set 2 approached and exceeded the value of Set 1, monkeys 
were increasingly likely to switch from Set 1 to Set 2 (Cumulative Gaussian goodness-of-fit tests; Average $\mathrm{R}^{2}=0.90$; Monkey $1: \mathrm{R}^{2}=0.91$; Monkey $2: \mathrm{R}^{2}=0.82$ ). Importantly, the $\mathrm{x}$ axis in Figure 3 does not show the absolute value of Set 2 but rather shows the relative value of Set 2 compared to Set 1 . This means that the difference in quantity between the two sets was driving their behavior as opposed to the absolute value of one set. This figure also shows that extreme differences in cardinality (high and low x-values) led to extreme differences in behavioral patterns, with virtually no switches when Set 1 was much larger than Set 2 and almost always switches when Set 2 was much larger than Set 1. This pattern is predicted by repeated mental comparisons of the quantities throughout the baiting.

The animals' probability of switching to Set 2 is constrained by their weber fraction $(\mathrm{w}=$ 0.86). To visualize the match between their behavior and psychophysical predictions based on weber fractions, we calculated the predicted probability that an animal with a 0.86 weber fraction would switch to Set 2 for each set size that was baited. We then analyzed these predictions by the difference in set sizes (see Supplementary Figure 2), as was done in Figure 3, in order to see what timecourse of switching weber ratios predict. The predicted switches at a weber of 0.86 account for the observed probabilities of switching $(R=0.97, p$ $<.001$; Supplementary Figure 2). The relation between the weber fraction and the probability of switching remains strong after controlling for the experiment-wide probability that Set 2 will be larger than Set 1 for each value of Set 1 (partial $R=0.84, p<.001$ ). These results support the conclusion that the animals continuously compared the quantities of the sets using approximate representations of quantity.

In addition to testing the probability of switching, we also analyzed the value of Set 2 at the point when the animals switched. The number of items in Set 2 at the point when the monkeys committed a mid-baiting switch was proportional to the total number of items in Set 1 (Figure 4). This finding reinforces the claim that the monkeys' switching behavior was based on a running comparison of quantity. Figure 4 shows that the number of items in Set 2 at the switch point was greater for larger values of Set 1 . This result is important because it shows that the influence of the relative values of the two sets on the animals' switch behavior is observed not only in the probability that the animal will switch but also in the value that the animals chooses as the switch point. The relation between the value of Set 1 and the switch point was observed for correct and incorrect trials but on incorrect trials, the average switch point was considerably lower. On average, including both correct and incorrect trials, monkeys switched from Set 1 to Set 2 in the middle of the baiting sequence when the value of Set 2 was -1.4 items compared to Set 1 (Switch Point minus Set 1: Monkey $1=-1.5$; Monkey $2=-1.3$ ). Thus, the monkeys switched to Set 2 when it was approximately equal to Set 1 .

To rule out the possibility that subconscious cueing by the experimenter was influencing the animals' behavior, we conducted a control condition. In the control condition, the monkeys were tested by two experimenters, each of whom baited one of the two caches. Each experimenter was blind to the quantity of food items baited into the other's cache and so was unaware of which cache contained the larger quantity. This ensured that the human experimenters could not give subconscious cues to the correct choice because they did not know which cache was correct. Both monkeys performed significantly above chance on the 
first session of testing on the control condition (Mean Accuracy $=75 \%, \mathrm{p}<.05$; Monkey 1 : 19/24, $\mathrm{p}<.01$; Monkey 2: 17/24, $\mathrm{p}<.05$ ). Each animal was then tested on additional sessions using this control procedure for approximately 120 trials. The additional sessions tested the numerical pairs from 1 to 8 . As in the main experiment, we found that both animals frequently switched to Set 2 before Set 2 was completely baited (38\% of trials) and were more likely to switch to Set 2 as its value increased relative to Set 1 (Cumulative Gaussian goodness-of-fit tests; Monkey $1: \mathrm{R}^{2}=0.92$, Monkey 2: $\mathrm{R}^{2}=0.94$ ). Thus, the animals exhibited quantity-dependent switch behavior even when the experimenters were naïve to the quantities presented and unable to provide cues to the larger value.

We conducted an additional control condition for baiting-time to test the possibility that the animals discriminated the two sets on the basis of total duration of set presentation, instead of the number of items in each set. This duration control condition presented one set on each trial for a total duration of 30s, which always was longer than the baiting duration of the second set. Half of the trials presented the larger set for 30s whereas the remaining half of trials presented the smaller set for 30s. The animals were tested on two additional sessions with a 50/50 mix of these duration controls and the standard experiment trials. Monkeys performed above chance on the duration control trials (Mean Accuracy $=71 \%, \mathrm{p}<.05$; Monkey $1=16 / 24, \mathrm{p}=.07$; Monkey $2=18 / 24, \mathrm{p}<.05$ ) and there was no significant difference in performance between these control trials and the standard experiment trials (Standard trials $79 \%$ correct, Control Trials $71 \%$ correct $\left[\mathrm{chi}^{2}\right] \mathrm{N}=96, \mathrm{p}=.35$ ). The animals did not have a bias toward picking the cache with the longer baiting time (Cache with 30s baiting-time chosen $44 \%$ of trials). This suggests that the monkeys quantified the items in each cache and not the duration of presentation. Again, the animals exhibited spontaneous switching during the timing control trials (55\% of trials) and they were more likely to switch to Set 2 as its value increased relative to Set 1 (Cumulative Gaussian goodness-of-fit tests; Monkey $1: \mathrm{R}^{2}=0.95$, Monkey 2: $\left.\mathrm{R}^{2}=0.94\right)$.

The results from the main experiment and control conditions indicate that monkey switching behavior is best described by monkeys' iteratively comparing quantities and not by experimenter cueing or monkey sensitivity to baiting duration. The data are consistent with the conclusion that the monkeys sequentially compared the value of the first set to the itemby-item changes in the value of the second set. This type of 'increment and compare' algorithm is an instance of a condition-controlled loop. Every time an item was added to Set 2, the monkeys 1) incremented their mental representation of the value of Set 2 and, 2) executed a mental comparison between Set 1 and Set 2, until Set 2 was greater than or equal to Set 1 . At the point when Set 2 was approximately equal to or greater than Set 1, the monkeys terminated the comparison routine and committed to choose Set 2 . Thus, the animals loop through iterations of incrementing and comparing their numerical representations until the second set is equal to or greater than the first set, then they decide to choose the second set. If that condition is not met, they default to choose Set 1 . Because the quantitative representations that monkeys are using as the basis of this algorithm are approximate, as indicated by the numerical ratio effects shown in Figure 2, their ability to judge the relative values of the two sets is noisy and thus their switch points are only approximately related to the relative values of the sets. 
We used a Bayesian data analysis to formalize and test our explanation of the monkey's switching behavior as a noisy "increment and compare" algorithm. This data analysis technique allows us to specify a space of algorithms the monkeys could have used, but leave the particular algorithm to be determined from the behavioral data itself: some settings of the model parameters correspond to alternative accounts of the animal's cognitive processes (e.g. ones that don't "count"). By inferring the most likely combination of parameters from the behavioral data, we are able to provide statistical evidence for or against each of these alternatives. Our work builds off of recent applications of Bayesian data analysis to numerical cognition tasks in children (Lee \& Sanecka, 2011; Sarnecka \& Lee, 2009).

For the model, we assumed that the animals represented the value of Set 1 as an approximate quantity with scalar variability. For each item added to Set 2 , they (noisily) increment an approximate mental counter, compare the value of this counter to that for Set 1, and tend to switch if Set 2 contains more items. Each of these key steps was parameterized with a variable whose value was inferred from the data. These parameters include: (a) the variability of accumulators for Sets 1 and 2, (b) a baseline rate of switching, (c) a rate of switching when Set 2 has more elements than Set 1, (d) the probability of incrementing Set 2 when each element is added, and (e) a baseline attentional probability specifying how often monkeys ignore entire trials. Some settings of these parameters will lead to viable alternative algorithms that do not count and compare as we hypothesize. For instance, if the probability (d) of incrementing the set is found to be close to zero, monkeys are not updating representations of quantity with each item. If the data suggest that monkeys tend to switch with high baseline probability (b), their behavior is not dependent on the relative quantities of the two sets, and depends perhaps only on time. If Set 1 and 2 (a) are given very different noise (Weber ratio) values, monkeys may be using qualitatively different systems to represent each set. If monkeys precisely enumerate the quantities, the analysis will recover Weber ratios (a) that approach zero. Exacting counting is therefore a particular setting of the model parameters that could be supported by the data. Thus, by determining the parameter values that are most consistent with the behavioral responses, we effectively test these alternative accounts against the parameter values implied by the increment and compare algorithm.

We used a standard sampling approach to find the likely range of parameters (a)-(e) given the observed behavioral data. The model trades off between parameter values that occur in a likely a priori range, with those that fit the data well. We chose simple and standard forms for the priors and likelihood. The variability parameters (a) were given $\operatorname{Gamma}(2,1)$ priors on the variance. A Beta $(1,9)$ prior was chosen for $(b)$, corresponding to a low baseline expectation for switches. The probability of switching when Set 2 has more elements (c), incrementing Set $2(\mathrm{~d})$, and attending to a trial (e) were each given uniform $(\operatorname{Beta}(1,1))$ priors, corresponding to no initial biases for particular values. This makes their inferred values closely track the behavioral data. A Bernoulli likelihood was used to determine the probability of having switched at each baited item. For simplicity, the outcome--whether or not an animal switched--was treated as independent on each baited item, conditioned on the parameters and observed set items. Where possible, intermediate variables were integrated out (collapsed). The model was run using PyMC (Patil, Huard, \& Hauser, 2007) to sample values from the posterior distribution on these parameters, given the behavioral data. 
Markov-Chain Monte-Carlo was run for 500k steps, drawing a sample every 200 steps. The model was tuned for 50k steps using PyMC 's defaults, and run for 150k steps of burn-in. The quality of the model's inference was assessed using the standard method of running multiple chains from different starting positions. This revealed quantitatively similar results between chains. Code is available under the GNU public license from S.P. An introduction to these Bayesian analysis methods can be found in Kruschke (2010) and Gelman et al. (2013).

The Bayesian analysis yields a posterior distribution on each variable, quantifying how strongly we should believe each possible parameter value is the true one given how the animals behaved in the experiment. Figure 5 shows these posterior distributions and reveals parameter values consistent with the increment and compare algorithm: monkeys are found to have very high probabilities of incrementing with each additional element of Set 2 (Figure 5d). Monkeys show low baseline probabilities of switching (Figure 5b), and high probabilities of switching when they believe that Set 2 contains more elements (Figure 5c). As such, their behavior is statistically consistent with sequential updating of Set 2, combined with decisions to switch based on approximate cardinality comparison. The model recovers similar weber fractions from the monkeys' switch trials (Set $1=0.89$, Set $2=0.64$ for Monkey 1 ; Set $1=0.81$, Set $2=0.82$ for Monkey 2 ) to the .86 weber fraction simply fit in monkeys' performance across all trials, helping to validate the general approach (Figure 5a $\& 5 b$ ). This is true even though ratios for Set 1 and Set 2 are treated independently by the analysis. Indeed, both weber fractions indicate considerable variability, consistent with nonexact representations. The analysis also reveals a moderately high probability of attending on each trial, meaning that some behavioral noise may plausibly represent failures to attend (Figure 5e).

We also tested whether this model of quantitative processing accounted for the monkeys' behavior on each control condition. The posterior distributions of all variables derived from the control data replicated qualitatively and quantitatively those derived from the main experiment data (Supplementary Figure 1). The posterior distribution in each control condition had similar ranges and modes to the full analysis on all key variables, with some small numerical differences observed in probability of attention on each trial. Most critically, both control conditions showed a probability of incrementing close to 1.0 and Weber ratios consistent with approximate representations of both sets. In general, these analyses confirm that the monkeys' quantitative switching behavior during the control conditions also was consistent with an 'increment and compare' algorithm.

In general, these results show that the monkeys' behavior is consistent with an increment and compare algorithm, across both the experimental and control conditions, and that alternative accounts - corresponding to particular settings of the data analysis model's parameters -are not supported.

\section{Discussion}

Our paper offers 1) a novel observation of spontaneous incremental quantity processing in non-human primates, 2) a novel theoretical contribution in the formalization of a cognitive 
algorithm underlying non-human quantification that parallel principles inherent in human counting, and 3) a novel methodological contribution in the implementation of a Bayesian analysis technique to test the nonverbal counting model against the primate data.

Although many studies have examined the capacity of non-human animals for quantitative representation, there has been no formal proposal of the cognitive algorithm that animals use to compare quantitative representations. The dominant nonverbal quantity model proposed by Gibbon, Meck, \& Church (Gibbon, Meck, \& Church, 1984; Meck \& Church, 1983) is mostly implemented as a conceptual model. In that model, animals only compare quantities after representations are entered into memory - there is no cognitive pathway for updating or comparing representations item-by-item. We found that monkeys spontaneously compared a given quantitative value to item-by-item changes in a second value. The data show that non-human primates inherently increment and compare quantitative values using a type of sequential logic observed in human counting (Gallistel \& Gelman, 1992; Carey, 2001; Dehaene, 1997). In human counting, an internal counter is sequentially incremented for each item in a set until some condition is met (eg., a target number is reached, all items are counted, or one set is greater than another). Our data indicate that non-human primates have the ability to use this type of condition-controlled quantitative logic to make sequential non-symbolic judgments of quantitative values. Moreover, our data show that animals used this quantitative comparison process spontaneously in that their switching behavior was not required by the task. The control condition data show that the animals did not rely on experimenter cueing or the temporal duration of the sequences to make quantity choices. The animals' spontaneous switching behavior during quantitative decision-making provides insight into the cognitive algorithm underlying their choices. By capitalizing on the monkeys' spontaneous switching behavior, we formalized a proposal of the cognitive algorithm that animals used to compare quantitative representations. We tested an 'increment and compare' mechanism in a Bayesian model analysis. This novel approach is powerful because it provides a theory-driven statistical test of the whole parameter space associated with our cognitive model. The 'increment and compare' algorithm that we tested predicted that the animals noisily increment an internal counter for each item presented in a set and then compare each update in value against their internal representation of a comparison value. The model results show that these parameters account for the animals' spontaneous switching behaviors and quantitative choices. Alternative explanations of the animals' behavior including an inability to accurately approximate quantity, an absence of Weber's law, an inability to compare item-by-item changes in quantity, or random switching would have resulted in the Bayesian model arriving at particular parameter values. The analysis instead reveals that such parameter values were not supported by the behavioral data, but those implementing an 'increment and compare' algorithm were. The increment and compare algorithm accounted for animals' quantity choices across all conditions of the experiment, including the timing and cueing control conditions. The model results provide strong statistical evidence that an 'increment and compare' algorithm underlies the observed behavioral data.

Our results provide novel evidence of a sequential comparison algorithm available to nonhuman animals, but have not yet explored which perceptual dimensions this capacity relies on. In particular, the types of inputs that monkeys use to incrementally compare quantities 
could be numerical, spatial, or a combination of the two. Animals likely use a combination of numerical and volumetric properties of objects in order to quantify sets of food items (Stevens, Wood, \& Hauser, 2007). In humans, numerical representations have been shown to be functionally dependent on spatial representations from evidence of parallel numerical and spatial impairments in patients with parietal cortex damage (Zorzi, Priftis, Umilta, 2002). Thus, the computations underlying judgments of spatial extent bear a close cognitive and neural relationship with those of numerical judgments in humans. Current theories suggest that human mathematical concepts are evolutionarily and developmentally derived from the cognitive and neural mechanisms of spatial processing (Dehaene \& Cohen, 2007). We hypothesize that the sequential comparison algorithm reported here is an important computational precursor to the emergence of human counting, regardless of whether it is specific to numerical judgments or operates more generally over spatial quantities such as surface area or volume. The connection between this algorithm and counting is in the way in which representations of quantity are processed and updated sequentially, rather than the inputs that the algorithm takes.

Our data provide evidence that non-human primates use a type of sequential logic to compare quantities in sequence that is algorithmically and logically similar to human counting. Human counting requires incrementing, iteration, and condition-controlled logic. The counting-like algorithm that the monkeys used spontaneously in this task contains those logical elements. With each item added to a set, the algorithm increments a mental counter and performs an iteration of mental comparison. Then, if the condition is met that the second set is approximately equal to or greater than the first set, the algorithm commits to choose it by switching. These logical rules are inherent in human counting.

Previous research in developmental psychology has sought to identify relations between nonverbal quantity estimation and human counting. Gelman \& Gallistel (eg., 1978, 1992, 2000) relate specific principles of verbal counting to specific principles of nonverbal counting mechanisms. For example, they argue that a "stable order principle", in which the tags used to enumerate a set are ordered consistently, is observed both in children's verbal counting behavior and in the accumulator mechanism of Meck \& Church's nonverbal counting model from non-human animals (Meck \& Church, 1983). Gelman \& Gallistel propose that skeletal principles provide the scaffolding upon which verbal counting routines emerge and there is support for elements of this "first principles" theory (eg., Cantlon \& Brannon, 2006; Cantlon, Fink, Safford, \& Brannon, 2007; Gallistel \& Gelman, 1992, 2000; Geary, 1995; Gelman, 1990; Gelman \& Brenneman, 1994; Izard, Pica, Spelke, \& Dehaene, 2008; Mix, 2002; Spelke, 1994; Starkey, Spelke, \& Gelman, 1990). The types of principles that relate nonverbal quantification and human counting differ between our proposal and "first principles" proposals. However, our conclusions are consistent with the general claim that a set of core algorithmic operations which are rooted in nonverbal quantity representation form the basis of verbal counting.

Although there are structural similarities in the algorithms underlying non-human "counting" and human counting, human counting extends beyond the capacity of nonhuman primate counting because it includes a symbolic component wherein each item in a set is not just put into correspondence with an internal counter, but also with a term in the 
ordered list of number symbols (Dantzig, 1954) or, in the case of earlier humans, a slash on a stick or bone. Human counting also gives rise to conceptual inferences about the structure of numerical sequences, such as the successor function (Carey, 2001). Non-human primates do not have a formal symbolic system for labeling item-by-item changes in set size or making symbolic inferences about number sequences. The absence of a symbol system prevents non-human primates from composing a precise representation of quantity. Yet, our results indicate that non-human primates possess the cognitive operations that are logically necessary for sequentially tracking and comparing quantities item-by-item. Animals can use this counting-like logic in the absence of a symbolic system of number to make approximate comparisons of quantitative values in sequence. The fact that this quantitative logic is present in non-human primates indicates that it predated counting and tallying in our evolutionary history, and perhaps was a critical piece of cognition for the human invention of formal counting.

\section{Supplementary Material}

Refer to Web version on PubMed Central for supplementary material.

\section{Acknowledgments}

We thank Jenna Bovee, Louis DiVincenti, Jeb McConnell, and Jeff Wyatt for research support. Funding was provided by the James S. McDonnell Foundation (220020300), the Alfred P. Sloan Foundation (FG-BR2013-019), and NSF Education Core Research.

\section{References}

Agrillo C, Dadda M, Bisazza A. Quantity discrimination in female mosquitofish. Animal Cognition. 2007; 10(1):63-70. [PubMed: 16868736]

Barnard AM, Hughes KD, Gerhardt RR, DiVincenti L, Bovee JM, Cantlon JF. Inherently analog quantity representations in olive baboons. Frontiers in Comparative Psychology. 2013; 4(253):1-11.

Beran M. Summation and numerousness judgments of sequentially presented sets of items by chimpanzees (pan troglodytes). Journal of Comparative Psychology. 2001; 115(2):181-19. [PubMed: 11459165]

Beran MJ. Rhesus monkeys (macaca mulatta) enumerate large and small sequentially presented sets of items using analog numerical representations. Journal of Experimental Psychology-Animal Behavior Processes. 2007; 33(1):42-54. [PubMed: 17227194]

Beran MJ, McIntyre JM, Garland A, Evans TA. What counting for 'counting'? Chimpanzees respond appropriately to relevant and irrelevant information in a quantity judgment task. Animal Behaviour. 2014; 85:987-993. [PubMed: 23750039]

Boysen ST, Bernston GG. Numerical competence in a chimpanzee (Pan troglodytes). Journal of Comparative Psychology. 1989; 103(1):23-31. [PubMed: 2924529]

Brannon E. The development of ordinal numerical knowledge in infancy. Cognition. 2002; 83(3):223240. [PubMed: 11934402]

Brannon E, Terrace H. Ordering of the numerosities 1 to 9 by monkeys. Science. 1998; 282(5389): 746-749. [PubMed: 9784133]

Cantlon JF, Brannon EM. The effect of heterogeneity on numerical ordering in rhesus monkeys. Infancy. 2006; 9(2):173-189.

Cantlon J, Brannon E. Shared system for ordering small and large numbers in monkeys and humans. Psychological Science. 2006; 17(5):401-406. [PubMed: 16683927]

Cantlon JF, Brannon EM. Basic math in monkeys and college students. PLoS Biology. 2007; 5(12):e328. [PubMed: 18092890] 
Cantlon J, Fink R, Safford K, Brannon EM. Heterogeneity impairs numerical matching but not numerical ordering in preschool children. Developmental Science. 2007; 10(4):431-440. [PubMed: 17552933]

Carey S. Cognitive foundations of arithmetic: Evolution and ontogenesis. Mind \& Language. 2001; 16(1):37-55.

Dantzig, T. Number: The Language of Science. Macmillan; 1954.

Dehaene, S. The Number Sense: How the mind creates mathematics. Oxford: Oxford University Press; 1997.

Dehaene S. Origins of mathematical intuitions: The case of arithmetic. Ann NY Acad Sci. 2009; 1156:232-259. [PubMed: 19338511]

Dehaene S, Cohen L. Cultural recycling of cortical maps. Neuron. 2007; (56):384-398. [PubMed: 17964253]

Emmerton, J. Cook, RG., editor. [Accessed April 1, 2012] Birds' judgments of number and quantity. Avian Visual Cognition. 2001. Available at http://www.pigeon.psy.tufts.edu/avc/emmerton/

Ferster, CB.; Skinner, BF. Schedules of reinforcement. New York: Appleton-Century-Crofts; 1957.

Flombaum J, Junge J, Hauser M. Rhesus monkeys (Macaca mulatta) spontaneously compute addition operations over large numbers. Cognition. 2005; 97(3):315-325. [PubMed: 16260264]

Geary DC. Reflections of evolution and culture in children's cognition: Implications for mathematical development and instruction. American Psychologist. 1995; 50(1):24. [PubMed: 7872578]

Gallistel, C. The Organization of Learning. Bradford Press; 1989.

Gallistel C, Gelman R. Preverbal and verbal counting and computation. Cognition. 1992; 44(1-2):4374. [PubMed: 1511586]

Gallistel CR, Gelman R. Non-verbal numerical cognition: From reals to integers. Trends in Cognitive Sciences. 2000; 4:59-65. [PubMed: 10652523]

Gelman R, Brenneman K. 14 First principles can support both universal and culture-specific learning about number and music. Mapping the mind: Domain specificity in cognition and culture. 1994:369.

Gelman, A.; Carlin, JB.; Stern, HS.; Dunson, DB.; Vehtari, A.; Rubin, DB. Bayesian data analysis. CRC press; 2013.

Hauser M, Carey S, Hauser L. Spontaneous number representation in semi-free-ranging rhesus monkeys. Proceedings of the Royal Society: Biological Sciences. 2000; 267:829-833. [PubMed: 10819154]

Izard V, Pica P, Spelke ES, Dehaene S. Exact equality and successor function: Two key concepts on the path towards understanding exact numbers. Philosophical Psychology. 2008; 21(4):491-505. [PubMed: 20165569]

Jordan KE, MacLean E, Brannon EM. Monkeys match and tally quantities across senses. Cognition. 2008; 108:617-625. [PubMed: 18571636]

Kruschke, J. Doing Bayesian data analysis: A tutorial introduction with R. Academic Press; 2010.

Lee MD, Sarnecka BW. Number-knower levels in young children: Insights from Bayesian modeling. Cognition. 2011; 120(3):391-402. [PubMed: 21109239]

Lipton J, Spelke E. Origins of number sense: Large-number discrimination in human infants. Psychological Science. 2003; 14(5):396-401. [PubMed: 12930467]

Meck WH, Church RM. A mode control model of counting and timing processes. Journal of Experimental Psychology: Animal Behavior Processes. 1983; 9(3):320. [PubMed: 6886634]

McCrink K, Wynn K. Large-number addition and subtraction by 9 -month-old infants. Psychological Science. 2004; 15(11):776-781. [PubMed: 15482450]

Mechner F. Probability relations within response sequences under ratio reinforcement. Journal of the Experimental Analysis of Behavior. 1958; 1(2):109-121. [PubMed: 16811206]

Mix KS. The construction of number concepts. Cognitive Development. 2002; 17(3):1345-1363.

Nieder A, Miller EK. Coding of cognitive magnitude: Compressed scaling of numerical information in the primate prefrontal cortex. Neuron. 2003; 37:149-157. [PubMed: 12526780]

Patil, A.; Huard, D.; Fonnesbeck, CJ. PyMC: Bayesian stochastic modeling in Python. 2010. 
Pepperberg I. Grey parrot numerical competence: a review. Animal Cognition. 2006; 9:377-391. [PubMed: 16909236]

Pica P, Lemer C, Izard V, Dehaene S. Exact and approximate arithmetic in an Amazonian indigene group. Science. 2004; 306(5695):499-503. [PubMed: 15486303]

Sarnecka BW, Lee MD. Levels of number knowledge during early childhood. Journal of Experimental Child Psychology. 2009; 103(3):325-337. [PubMed: 19345956]

Spelke E. Initial knowledge: Six suggestions. Cognition. 1994; 50(1):431-445. [PubMed: 8039373]

Starkey P, Spelke ES, Gelman R. Numerical abstraction by human infants. Cognition. 1990; 36(2):97127. [PubMed: 2225757]

Stevens J, Wood J, Hauser MD. When quantity trumps number: Discrimination experiments in cottontop tamarins and common marmosets. Animal Cognition. 2007; 10:429-437. [PubMed: 17354004]

Tomonaga M, Matsuzawa T. Enumeration of briefly presented items by the chimpanzee (pan troglodytes) and humans (homo sapiens). Animal Learning \& Behavior. 2002; 30(2):143-157. [PubMed: 12141135]

Wynn K. Addition and subtraction by human infants. Nature. 1992; 358:749-750. [PubMed: 1508269]

Xu F, Spelke E. Large number discrimination in 6-month-old infants. Cognition. 2000; 74(1):B1-B11. [PubMed: 10594312]

Zorzi M, Priftis K, Umilta C. Neglect disrupts the mental number line. Nature. 2002; 417:138-139. [PubMed: 12000950] 
A) Cache 1 is sequentially baited with 5 food items

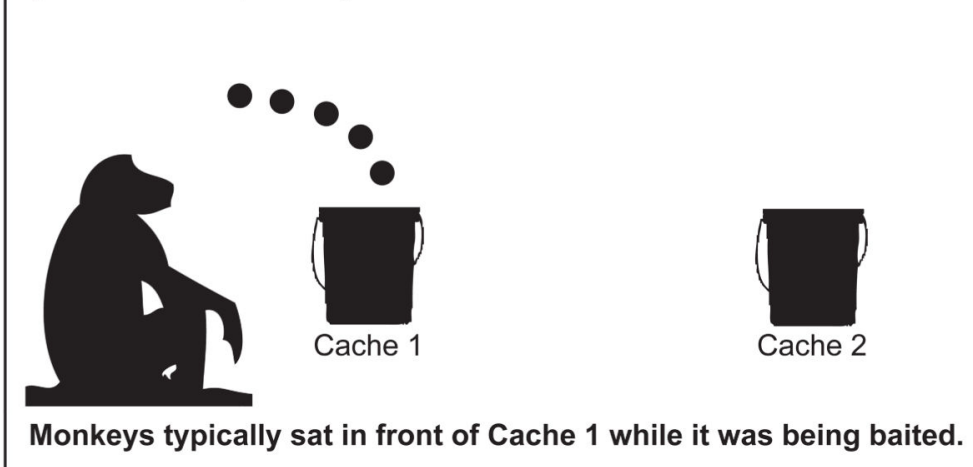

B) Then, Cache 2 is sequentially baited

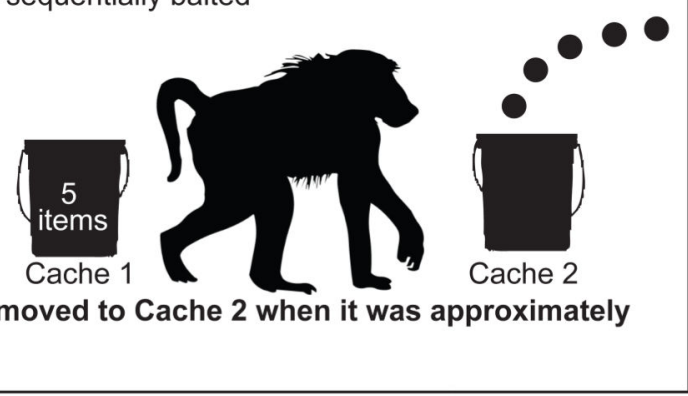

Figure 1.

Example Trial. Baboons were presented with a choice between two sequentially-presented sets of food items. We observed that they frequently committed to a choice after the first set was completely baited but before the second set was completely baited. The baboons indicated that they had reached an early decision by physically moving from the first set to the second set in the middle of a trial. 


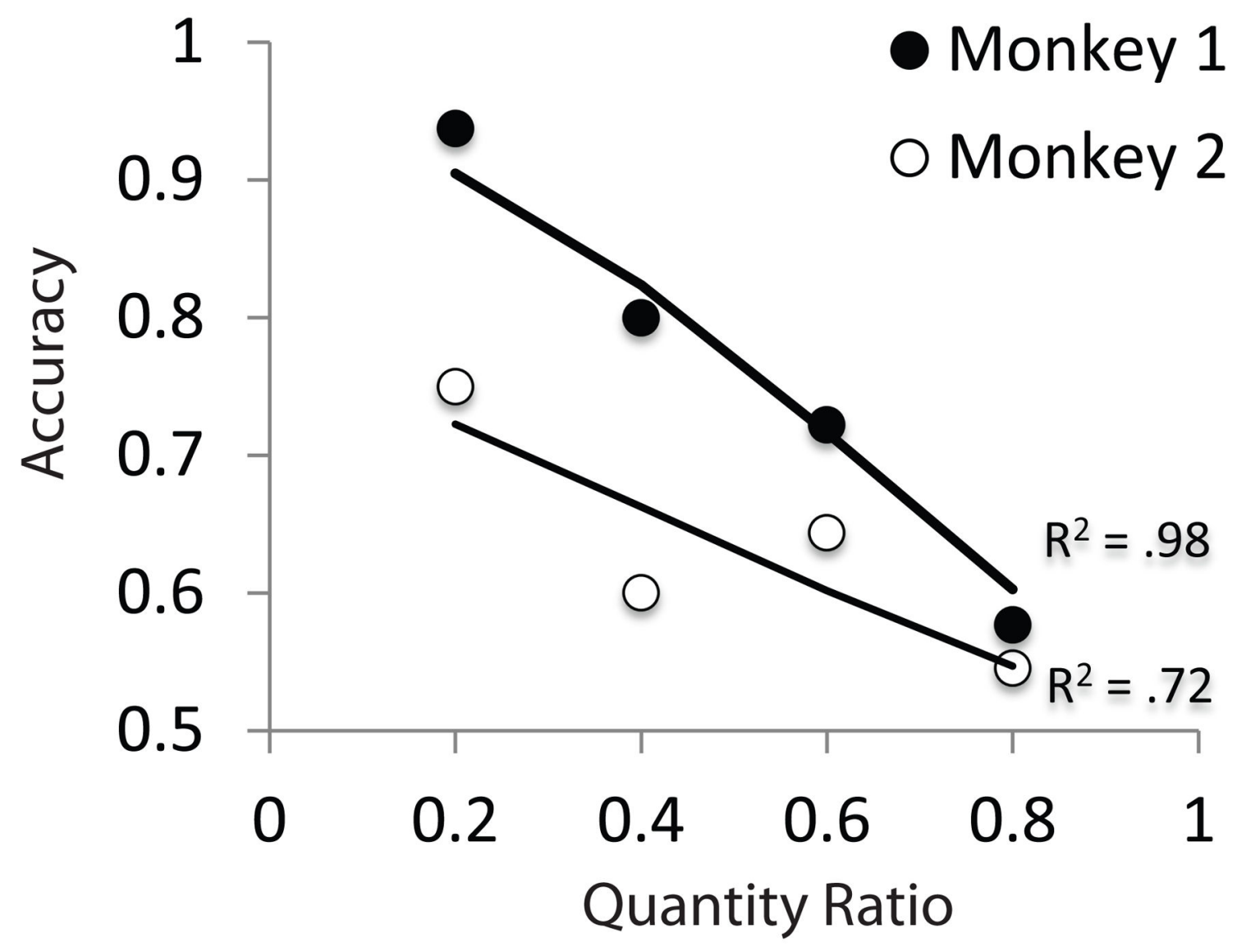

Figure 2.

Ratio Effects. Both monkeys showed evidence of approximate quantification in that they showed ratio effects in their ability to choose the larger quantity of food items. As the ratio between quantities increased (and became finer), monkeys' accuracy at choosing the larger quantity decreased. 


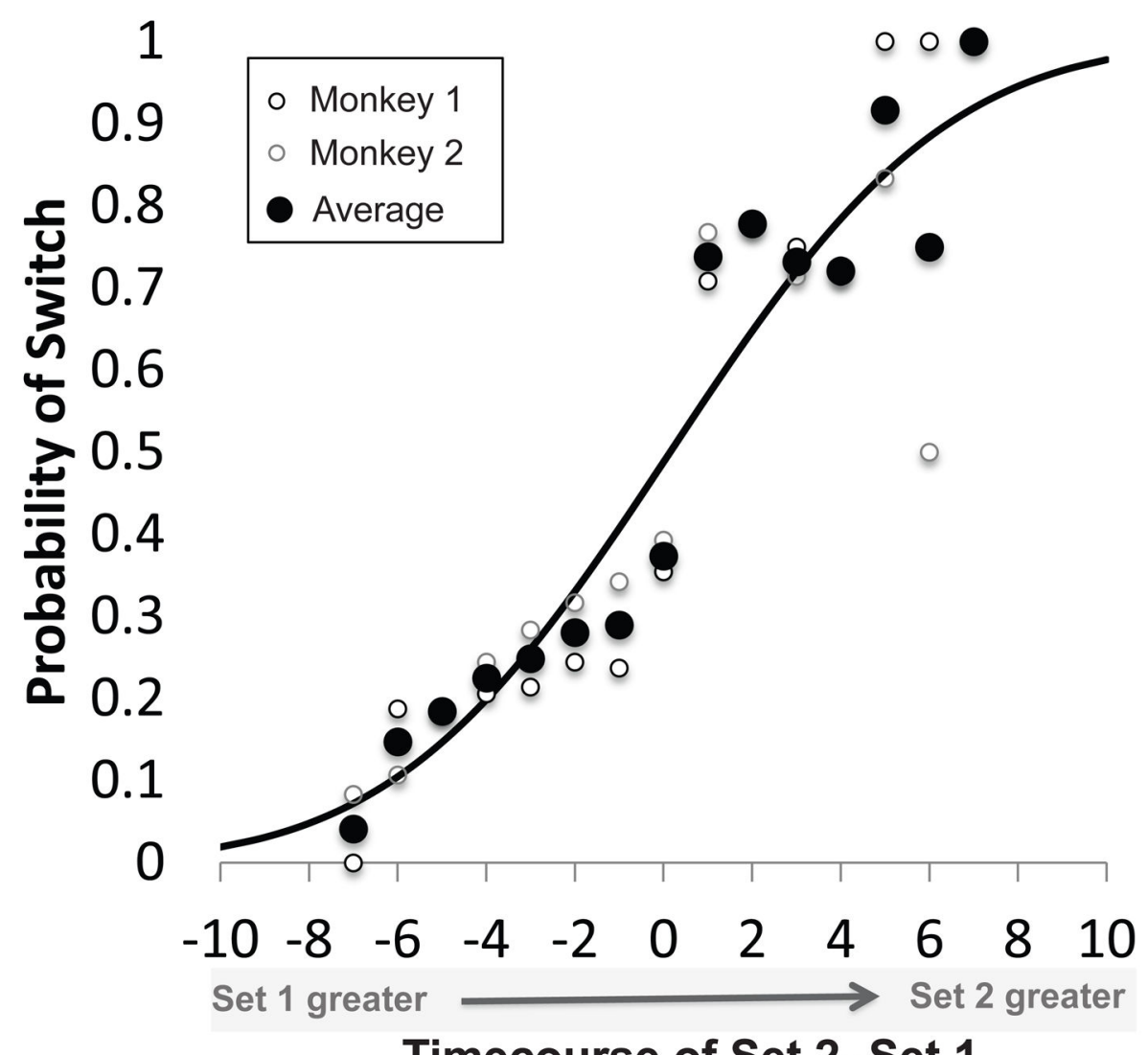

Timecourse of Set 2- Set 1

Figure 3.

Probability of a switch behavior. Monkeys were increasingly likely to switch to Set 2 as the difference between Set 1 and Set 2 approached zero and the value of Set 2 began to exceed that of Set 1 . 


\section{Switch Point}
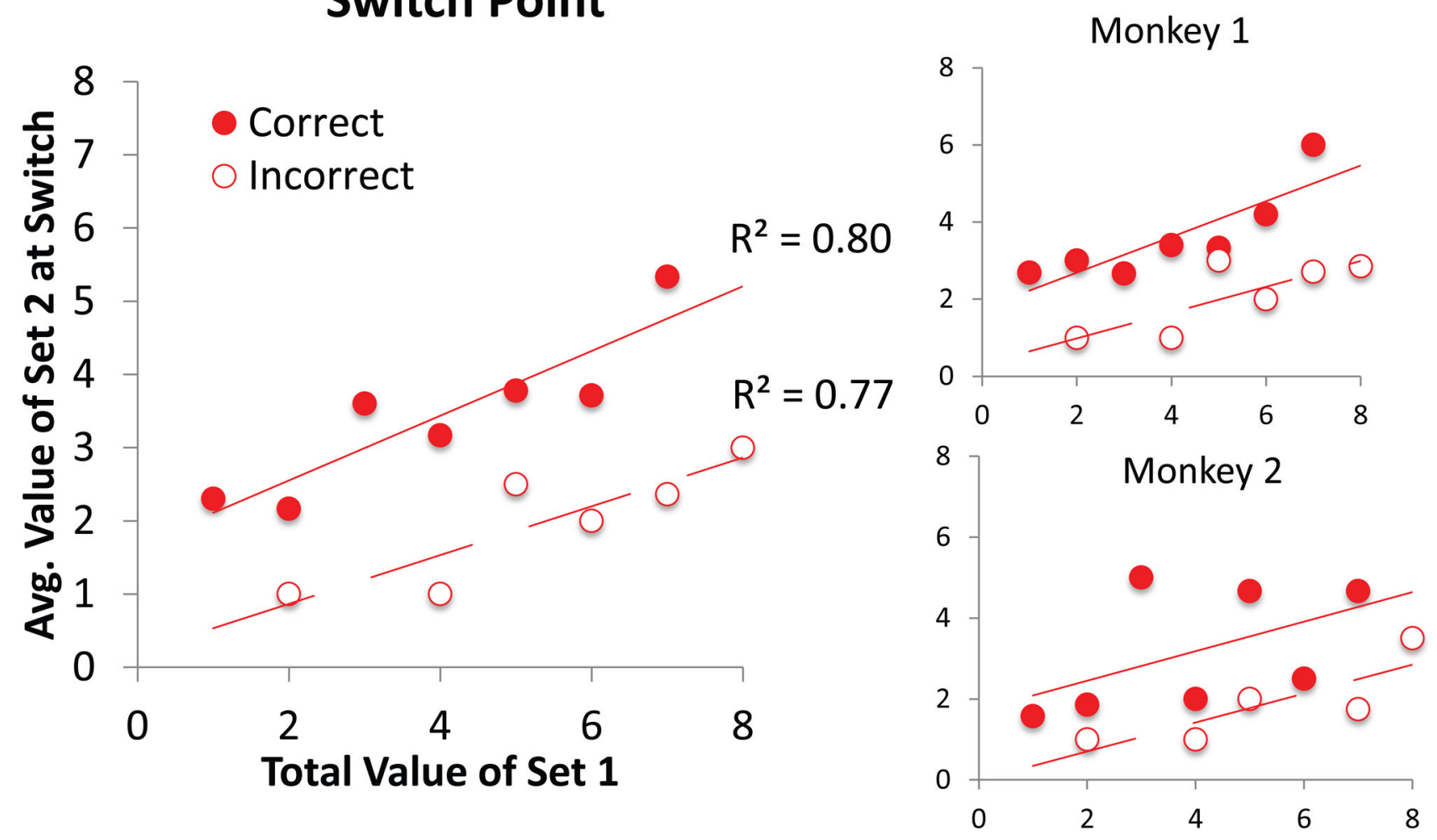

Figure 4.

Average Switch Point. The value of Set 2 at which monkeys typically executed a midbaiting switch behavior (the switch point) was correlated with the total value of Set 1. Monkeys exhibited larger switch points for larger values of Set 1. 

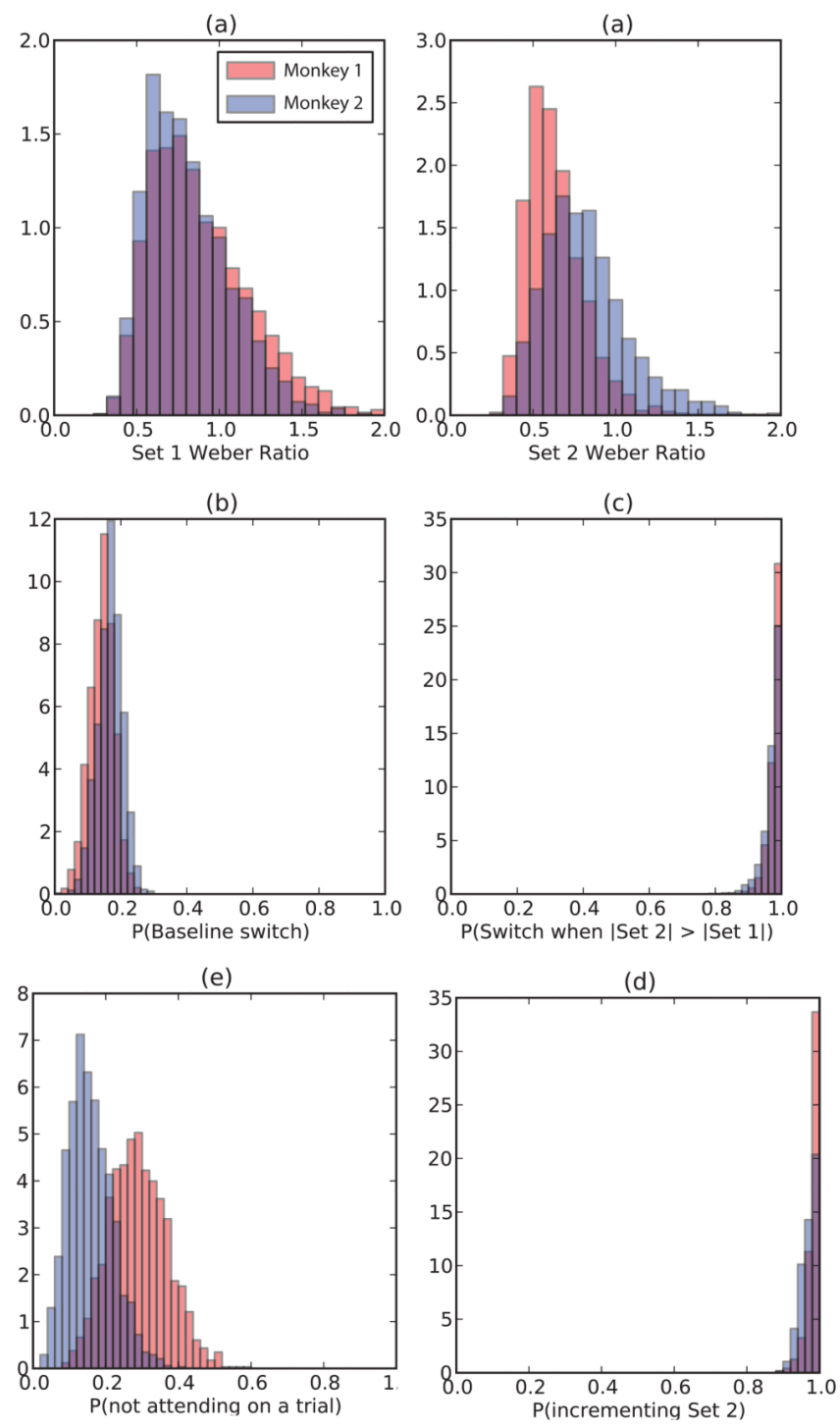

Figure 5 .

Posterior distributions on model parameters for a) the sensitivity of the accumulators for Set 1 (left) and Set 2 (right), measured by the Weber fraction, b) baseline probability of a switch, c) the probability of a switch when Set 2 was greater than Set 1, and d) the probability of attending to a trial. 